Supporting Information

\title{
High-strength carbon nanotube film from improving alignment and densification
}

Wei Xu†, Yun Chen¥, Hang Zhan† \& Jian Nong Wang†**

$†$ Nano Carbon Research Center, School of Mechanical and Power Engineering, East China

University of Science and Technology, 130 Meilong Road, Shanghai 200237, P. R. China.

$\ddagger$ School of Materials Science and Engineering, Shanghai Jiao Tong University, 800 Dong Chuan

Road, Shanghai 200240, P. R. China.

Correspondence to: jnwang@ecust.edu.cn 


\section{Experimental Methods}

1. Synthesis of Hollow Cylindrical CNT Assembly. The hollow cylinder-like CNT assembly was continuously synthesized at $1150-1300{ }^{\circ} \mathrm{C}$ in a horizontal furnace using an alumina tube with a diameter of $50 \mathrm{~mm}$ and nitrogen as the reactor and carrier gas, respectively. The precursor solution consisted of a liquid feedstock of carbon source (ethanol) with dissolved ferrocene (0.2-0.8 wt.\%) and thiophene (0.05-0.3 wt.\%). This solution was injected into the reactor at a rate of $2-10 \mathrm{ml} \mathrm{min}^{-1}$, and carried into the high-temperature zone by $\mathrm{N}_{2}$ at a flow rate of $10-30 \mathrm{~L} \mathrm{~h}^{-1}$. A film in the form of a cylinder with one end closed formed on the inner side wall of the reactor at the downstream, and was driven out from the reactor to air atmosphere by the enclosed $\mathrm{N}_{2}$ and gaseous products from the pyrolysis of the reaction solution.

2. Formation of CNT Film. A winding drum with a diameter of 0.4 or $1 \mathrm{~m}$ was used for winding CNTs. The winding drum was wrapped with an Al foil substrate which was wetted with ethanol. As soon as the CNT cylinder was introduced on the wet Al foil, it shrank to a film. Since the film was very sticky, it strongly stunk on the Al foil and could be winded on the drum as the drum rotated. The winding rate was 4-20 $\mathrm{m} \mathrm{min}^{-1}$, depending on the specific experimental conditions. The width of the $\mathrm{Al}$ substrate wrapped on the winding drum was preset. At a given substrate surface area, the thickness of the film was varied by supplying different amounts of reaction solution and thus depositing different amounts of CNT material.

For the preparation of the random CNT film, a small plate $(8 \mathrm{~cm} \times 8 \mathrm{~cm})$, which was also covered with Al foil, was fixed on the winding drum and put horizontally close the exit of the reactor. $\mathrm{X}$ direction was marked on the plate, parallel to the length of the tube reactor. The CNT cylinder was extruded from the reactor at the usual rate, equal to that used for making the slightly aligned sample, and deposited on the plate as the winding drum rotated. After some deposition, the plate was 
reoriented, relative to the length of the tube reactor, by $10^{\circ}$. Deposition of CNT cylinder on the plate resumed. This process was repeated at each $10^{\circ}$ interval until the $\mathrm{X}$ direction had rotated by $180^{\circ}$.

The CNT film was separated from the Al foil substrate by soaking in a dilute $\mathrm{HCl}$ acid. After washing and rinsing, the film was dried in a vacuum chamber at $60{ }^{\circ} \mathrm{C}$.

3. Mechanical Rolling of CNT Film. We applied conventional rolling to enhance the packing density of the CNTs in the film (ZK2015OR, Dongguan Chi Division Precision Machinery Co., Ltd., China) (Figure S7). In doing this, the top of the as-prepared film on the Al foil substrate was covered with another piece of $\mathrm{Al}$ foil. Such a sample was rolled between two rollers running at opposite directions. Before each rolling, the two rollers were such adjusted that they were in touch with each other and there was no gap between them from visualization of naked eyes. To improve rolling homogeneity, the same sample was rolled several times. See Video S3 for the rolling process.

4. Structure Characterization and Analysis. CNTs were characterized by thermo-gravimetric analysis (TGA, Netzsch Model STA 409 PC, heating rate of $10{ }^{\circ} \mathrm{C} \min ^{-1}$ and a constant air flow of $20 \mathrm{ml} \mathrm{min}{ }^{-1}$ ), high resolution transmission electron microscopy (HR-TEM, JEOL-2010F, accelerating voltage of $200 \mathrm{kV}$ ), scanning electron microscopy (SEM, FEI Nova NanoSEM 450, Holland), and Raman spectroscopy (Raman, Bruker Senterra R200-L, excitation wavelength of 532 $\mathrm{nm}$ ). Radial breathing mode (RBM) Raman spectrum was used to estimate the diameter of the CNTs using the relation $\omega_{\mathrm{r}}=6.5+223.75 / d_{\mathrm{r}}$, where $\omega_{\mathrm{r}}$ and $d_{\mathrm{r}}$ are the position of Raman shift and the diameter, respectively.

The degree of nanotube alignment in a CNT film was measured using polarized Raman spectroscopy. The intensities of the G-band were recorded when the incident laser beam was 
respectively placed parallel and perpendicular to the winding direction of the CNT film. The ratio of $\mathrm{I}_{\mathrm{G} / /} / \mathrm{I}_{\mathrm{G} \perp}$ was adopted in this work to describe the alignment degree of CNTs.

For thickness measurement by SEM, a CNT ribbon was vertically attached on the sample stage with conductive adhesive glued on two sides. The stage was carefully rotated until a clear cross section could be visualized. The samples used for thickness measurement were always directly cut from those that were to be used for tensile testing.

5. Tensile Testing. Tensile tests were performed on a fiber tensile tester, which is equipped with a deformation loading system and a force measuring system with a maximum force of $15 \mathrm{~N}$ and precision of $0.01 \mathrm{cN}$. The samples used for tensile measurement had a length of $30 \mathrm{~mm}$ and width of $2 \mathrm{~mm}$, and they were cut along the longitudinal direction (or the winding direction) of the film by a razor blade. The samples were directly fixed on the tester with clamps at both ends after the tester was calibrated. After the upper part of the sample had been clamped, a small weight (0.5 g) was applied on the lower end, to ensure that the sample was taut. Then, the lower part of the sample was clamped at the position to have a desired gauge length (GL). The tensile testing was performed at a displacement rate of $20 \mathrm{~mm} \mathrm{~min}^{-1}$ and a GL of $10 \mathrm{~mm}$ using ribbon-like fibers with a width of 2 $\mathrm{mm}$, which corresponds to an engineering strain rate of $\sim 3.33 \times 10^{-2} \mathrm{~s}^{-1}$. 


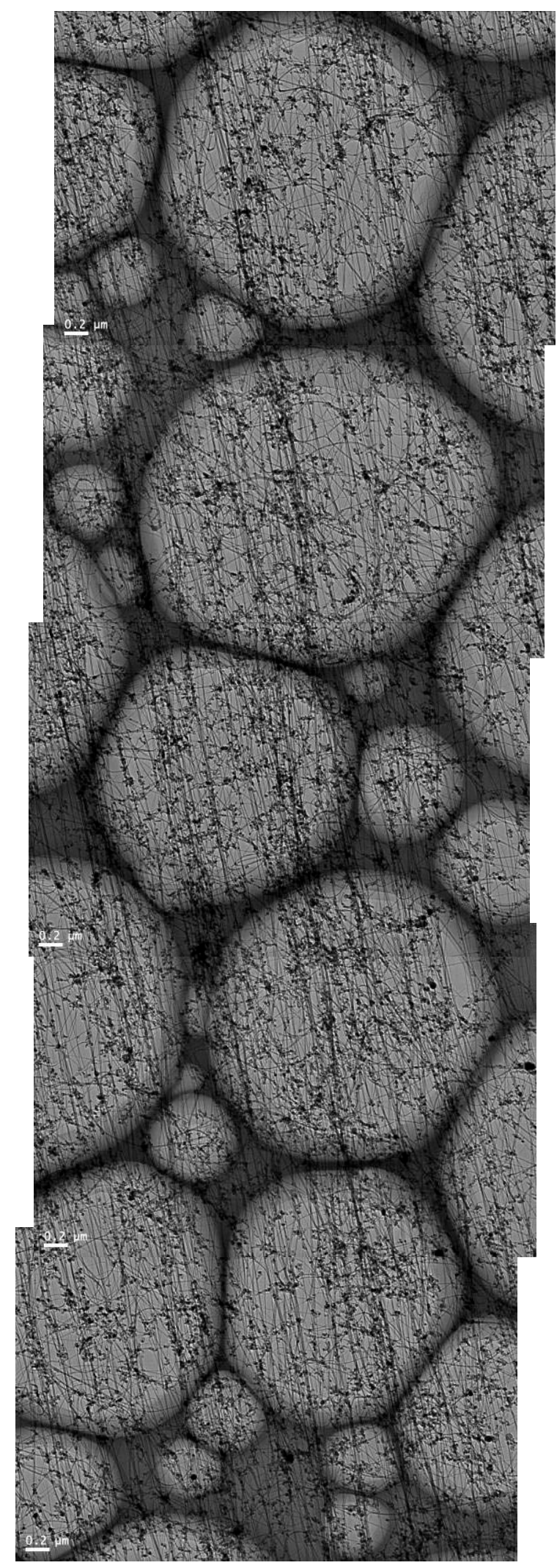

Figure S1. Extension of individual CNTs or CNT bundles with the attachment of Fe particles.

This figure contains a series of TEM images. To track the extension of individual CNT/bundles, it should be viewed at a high magnification. The TEM sample was prepared by picking up a segment of the CNT cylinder just coming out from the reactor and shrinking it on a Cu grid (TEM sample holder, $3 \mathrm{~mm}$ in diameter). 

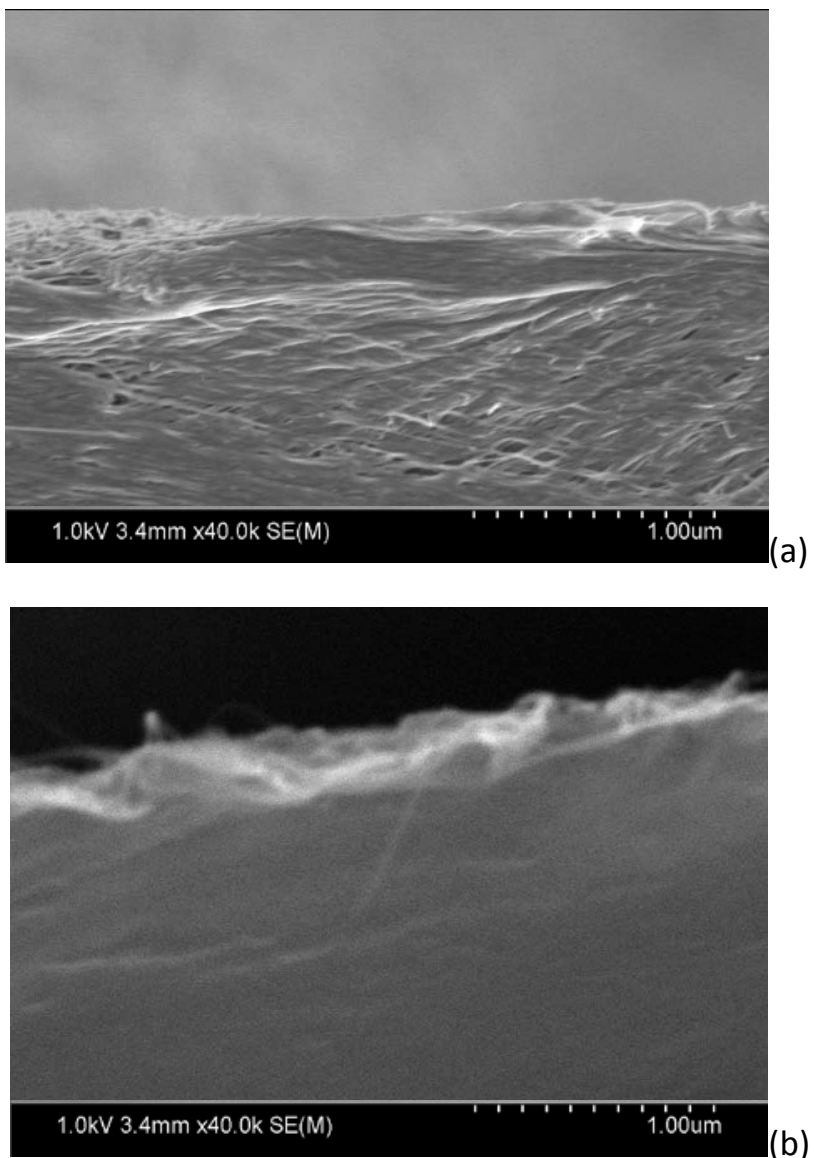

Figure S2. SEM images of the well aligned film surface before (a) and after rolling (b). 


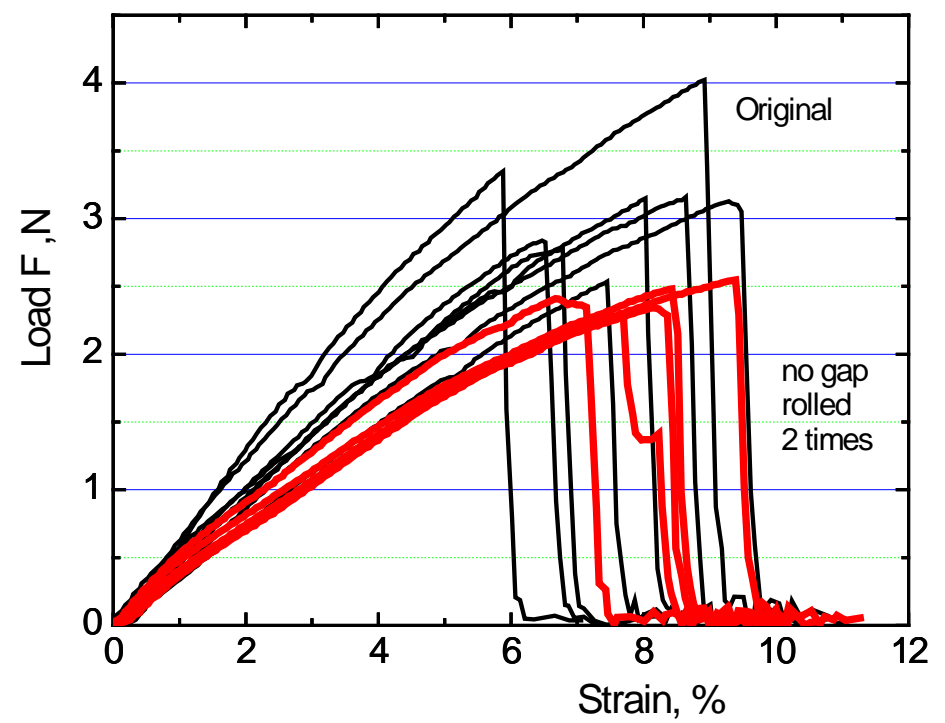

Figure S3. The load vs. strain curves for original and rolled sample (well aligned one). 

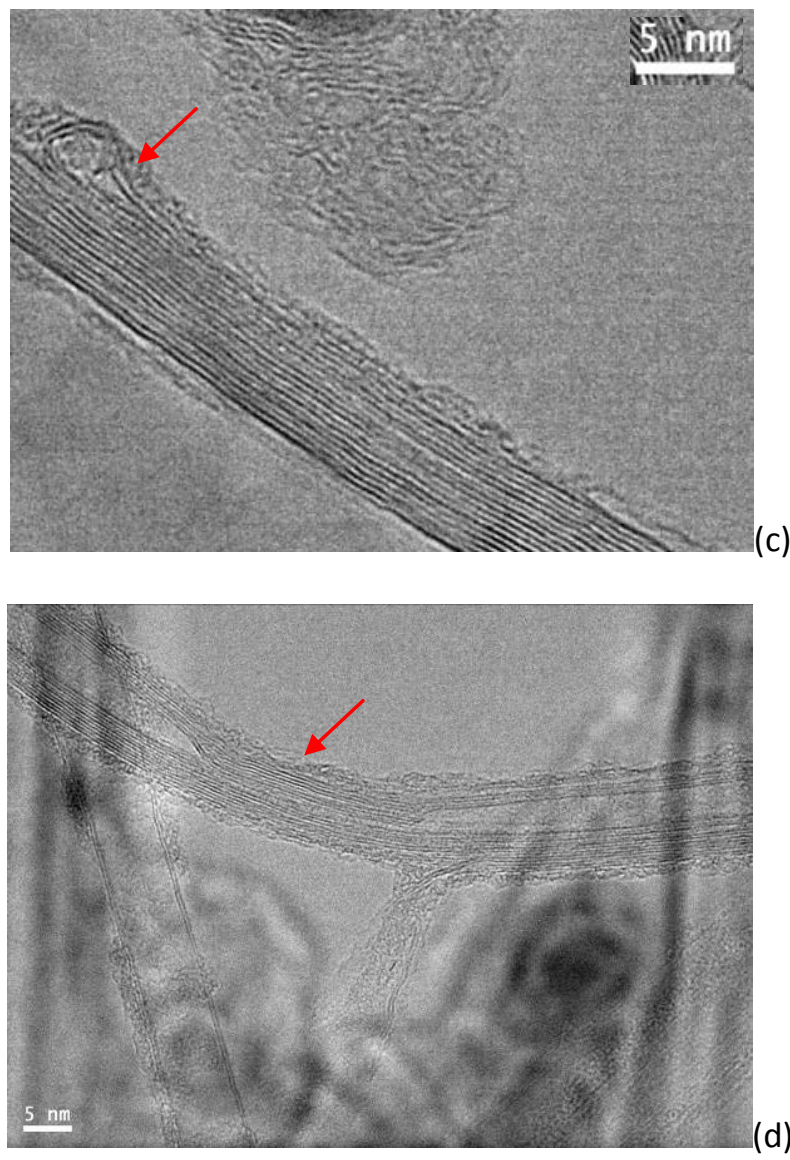

Figure S4. TEM images of collapsed double- and multi-walled CNTs sporadically observed in the unrolled film. Note the uncollapsed double-walled CNT with a diameter of $7.5 \mathrm{~nm}$ in (b). 


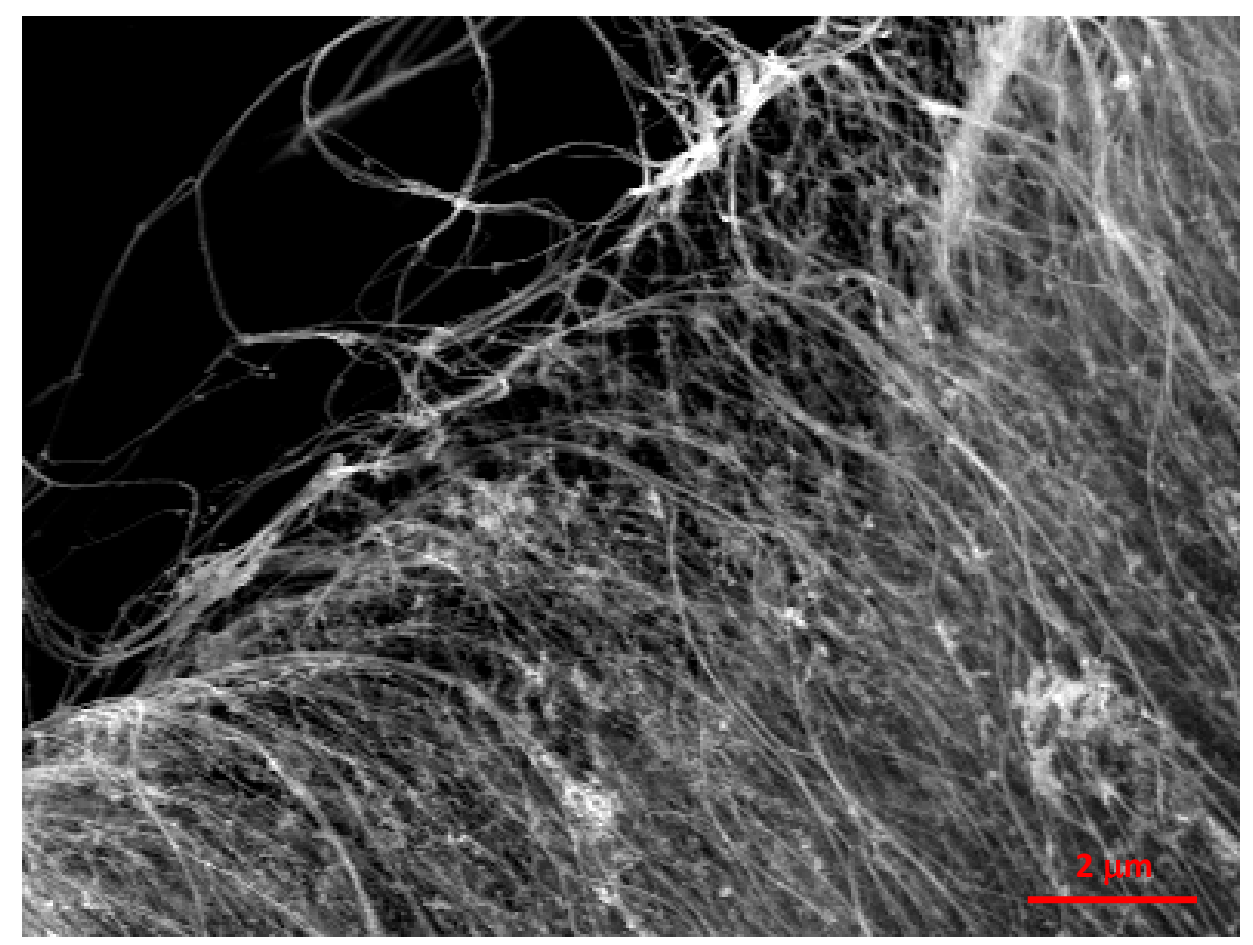

Figure S5. The fracture surface examined at high magnification. 

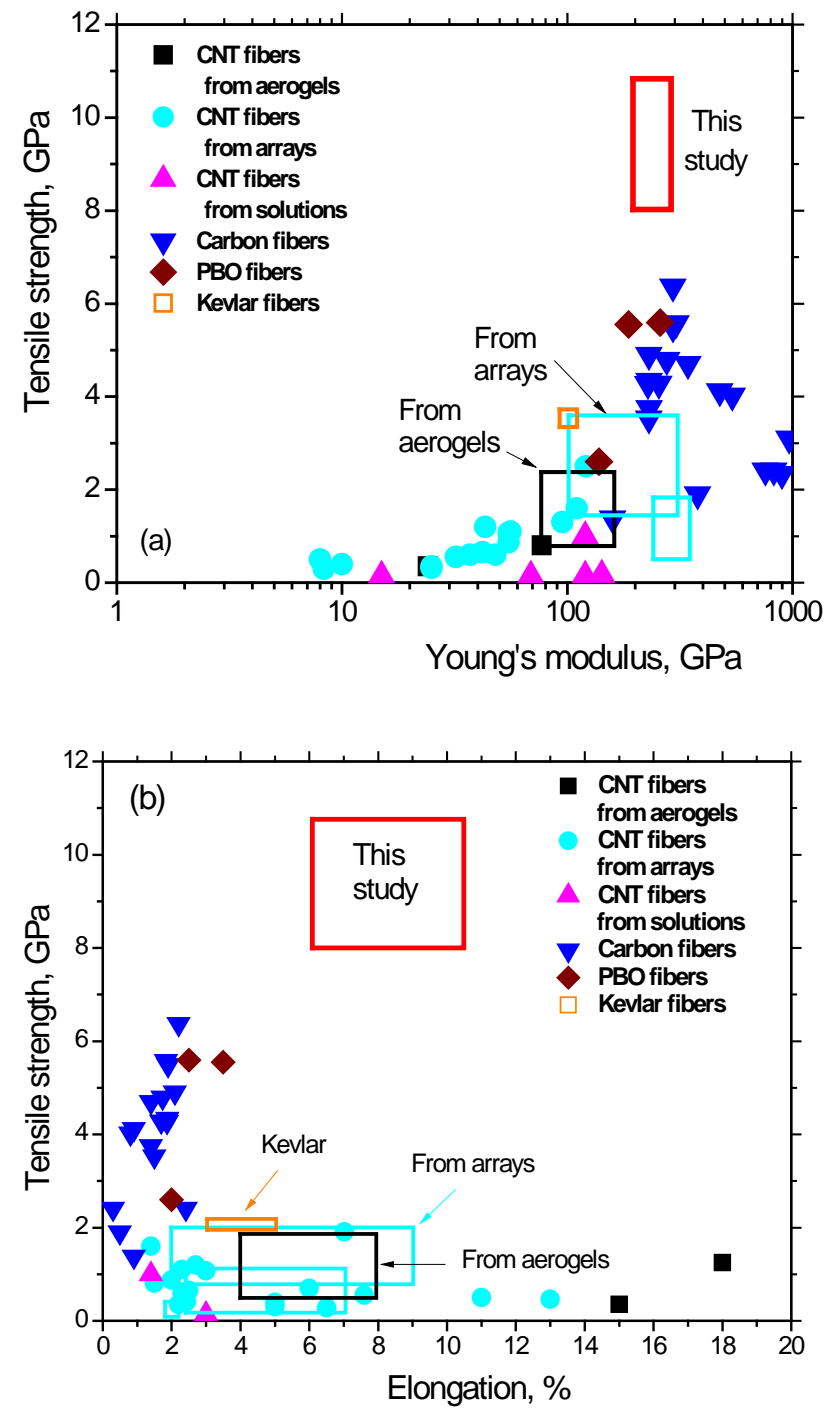

Figure S6. Comparison between the present CNT sample and previous fibers. (a) In the form of strength vs. Young's modulus. (b) In the form of strength vs. elongation. The strengths and moduli for CNT films are generally lower than those for fibers. Only results for previous fibers are included here for comparison. Data sources are listed below.

\section{CNT fibers from solutions:}

1. B. Vigolo, A. Penicaud, C. Coulon, C. Sauder, R. Pailler, C. Journet, P. Poulin, Macroscopic fibers and ribbons of oriented carbon nanotubes. Science 290, 1331-1334 (2000).

2. A. Dalton, S. Collins, E. Muñoz, J. Razal, V. Ebron, J. Ferraris, R. Baughman, Super-tough carbon-nanotube fibres. Nature 423, 703-703 (2003).

3. L. Ericson, H. Fan, H. Peng, V. Davis, W. Zhou, J. Sulpizio, R. Smalley, Macroscopic, neat, single-walled carbon nanotube fibers. Science 305, 1447-1450(2004).

4. N. Behabtu, C. Young, D. Tsentalovich, O. Kleinerman, X. Wang, A. Ma, M. Pasquali, Strong, light, multifunctional fibers of carbon nanotubes with ultrahigh conductivity. Science 339, 182-186 (2013).

From arrays:

5. K. Jiang, Q. Li, S. Fan, Nanotechnology: spinning continuous carbon nanotube yarns. Nature 419, 801-801 (2002). 
6. M. Zhang, K. Atkinson, R. Baughman, Multifunctional carbon nanotube yarns by downsizing an ancient technology. Science 306, 1358-1361(2004).

7. C. Tran, W. Humphries, S. Smith, C. Huynh, S. Lucas, Improving the tensile strength of carbon nanotube spun yarns using a modified spinning process. Carbon 47, 2662-2670 (2009).

8. K. Liu, Y. Sun, R. Zhou, H. Zhu, J. Wang, L. Liu, K. Jiang, Carbon nanotube yarns with high tensile strength made by a twisting and shrinking method. Nanotechnology 21, 045708 (2010).

9. X. Zhang, Q. Li, Y. Tu, Y. Li, J. Coulter, L. Zheng, Y. Zhao, Q. Jia, D. Peterson, Y. Zhu, Strong carbon-nanotube fibers spun from long carbon-nanotube arrays. Small 3, 244-248 (2007).

From aerogels (strength data from 1 and $2 \mathrm{~mm}$ gauge lengths are not included):

10. Y. Li, I. Kinloch, A. Windle, Direct spinning of carbon nanotube fibers from chemical vapor deposition synthesis. Science 304, 276-278 (2004).

11. K. Koziol, J. Vilatela, A. Moisala, M. Motta, P. Cunniff, M. Sennett, A. Windle, High-performance carbon nanotube fiber. Science 318, 1892-1895 (2007).

12. X. Zhong, Y. Li, Y. Liu, X. Qiao, Y. Feng, J. Liang, J. Li, Continuous multilayered carbon nanotube yarns. Adv. Mater. 22, 692-696 (2010).

Kevlar fibers:

13. C. Yue, G. Sui, H. Looi, Effects of heat treatment on the mechanical properties of Kevlar-29 fibre. Comp. Sci. Tech. 60, 421-427 (2000).

PBO fibers:

14. S. Kumar, T. Dang, F. Arnold, A. Bhattacharyya, B. Min, X. Zhang, P. Willis, Synthesis, Structure, and Properties of PBO/SWNT Composites. Macromolecules 35, 9039-9043 (2002).

15. T. Kitagawa, M. Ishitobi, K. Yabuki, An analysis of deformation process on poly- ${ }^{-}{ }^{-}$-phenylenebenzobisoxazole fiber and a structural study of the new high-modulus type PBO HM+ fiber. J. Polym. Sci. Part B: Polym. Phys. 38, 1605-1611 (2000).

Carbon fibers:

16. M. Minus, S. Kumar, The processing, properties, and structure of carbon fibers. JOM. 57, 52-58 (2005). 


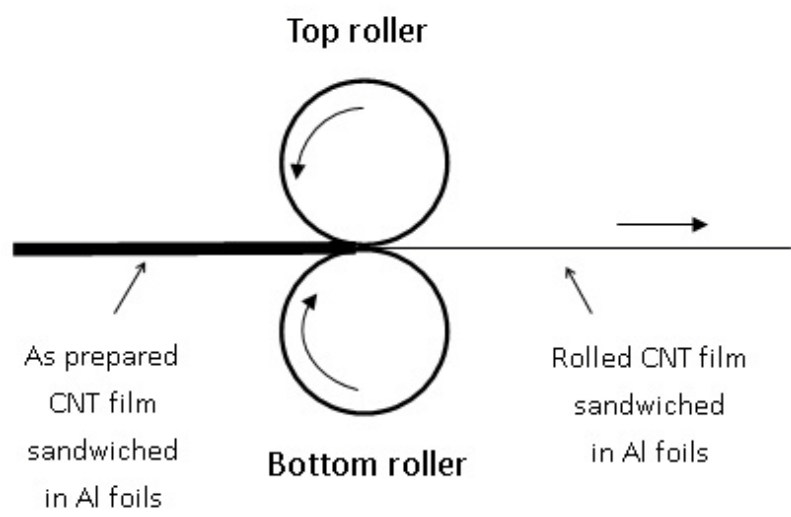

(a)

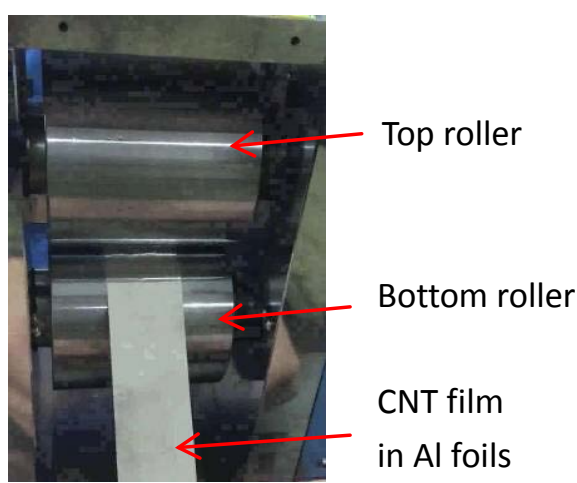

(b)

Figure S7. (a) Schematic illustration of the rolling system (side view). (b) The front view of the rolling system. The CNT film was sandwiched in Al foils and fed into the rollers. The rollers rotates, takes in the film, and rolls the film to a smaller thickness. See Video S3 for the rolling process. 